\title{
A Parametric Model for the Distribution of the Angle of Arrival and the Associated Correlation Function and Power Spectrum at the Mobile Station
}

\author{
Ali Abdi ${ }^{1}$, Janet A. Barger ${ }^{2}$, and Mostafa Kaveh ${ }^{3}$ \\ ${ }^{1}$ Dept. of Elec. and Comp. Eng., New Jersey Institute of Technology \\ ${ }^{2}$ Dept. of Elec. Eng., University of Texas at Tyler \\ ${ }^{3}$ Dept. of Elec. and Comp. Eng., University of Minnesota
}

Abstract _ One of the main assumptions in the Clarke's classic channel model is isotropic scattering, i.e. uniform distribution for the angle of arrival of multipath components at the mobile station. However, in many mobile radio channels we encounter non-isotropic scattering, which strongly affects the correlation function and power spectrum of the complex envelope at the mobile receiver. In this contribution, we propose the use of the versatile von Mises angular distribution, which includes and/or closely approximates important distributions like uniform, impulse, cardioid, Gaussian, and wrapped Gaussian, for modeling the non-uniform angle of arrivals at the mobile. Based on this distribution, associated correlation function and power spectrum of the complex envelope at the mobile receiver are derived. The utility of the new results is demonstrated by comparison with the correlation function estimates of measured data.

Point of contact: Ali Abdi

Dept. of Electrical and Computer Engineering New Jersey Institute of Technology

323 King Blvd.

Newark, NJ 07102, USA

Phone: (973) 5965621

Fax: (973) 5965680

Email: abdi@adm.njit.edu

The work of the first and the third authors have been supported in part by the National Science Foundation, under the Wireless Initiative Program, Grant \#9979443. 


\section{INTRODUCTION}

The received signal correlation functions and power spectra at the mobile station (MS) depend on the probability density function (PDF) of the angle of arrival (AOA) of a scattered wave. Clarke's twodimensional isotropic scattering model (a uniform AOA PDF over $[-\pi, \pi)$ ) gives rise to the zero-order Bessel function for the autocorrelation function and the U-shaped power spectrum for the complex envelope of multipath components at the MS [1, p. 40-43]. However, it has been argued [2]-[6], and experimentally demonstrated [7]-[16] that the scattering encountered in many environments is nonisotropic, resulting in a non-uniform PDF for AOA at the MS. As has been discussed in [16], the assumption of a uniform PDF for the AOA introduces small errors on the first order statistics of the received signal, but a significant error on the second order statistics, like correlation functions and level crossing rates.

In [17] and [18], two geometrically based angle of arrival PDF's are derived; while three different models are assumed for such a PDF by other authors: quadratic PDF [19], Laplace PDF [10], and cosine PDF [20]. In addition to showing good fit to measurements, another important factor in selecting a candidate for the angle of arrival PDF is the mathematical convenience it provides to the determination of closed-form solutions for the correlation functions, power spectra, spectral moments, etc.. The previously mentioned PDF's do not satisfy the latter requirement. Achieving this goal is the main purpose of this paper.

The remainder of the paper is organized as follows. In Section II we present the two parameter von Mises PDF as flexible model for the PDF of the angle of arrivals. The utility of this model is demonstrated by the derivation of closed-form expressions for the correlation function and power spectrum of the complex envelope at the MS in Section III. This is followed by a study of the differences in the characteristics of the correlation function and power spectrum for cases which deviate from the isotropic scattering model introduced by Clarke. Finally, measured data is used to confirm the validity of the proposed model in predicting the scattering characteristics encountered in actual mobile communication situations. 


\section{A FLEXible PDF FOR THE AOA}

A PDF was introduced by R. von Mises in 1918 to study the deviations of measured atomic weights from integral values [35]. This PDF plays a prominent role in statistical modeling and analysis of angular variables [21, pp. 57-68]. Let the random variable $\Theta$ represent the AOA of a multipath component (scatter and specular), received at the MS. The von Mises PDF for the scatter component of $\Theta, p_{\Theta}^{\text {scat }}(\theta)$, is given by:

$p_{\Theta}^{\text {scat }}(\theta)=\frac{\exp \left[\kappa \cos \left(\theta-\theta_{p}\right)\right]}{2 \pi I_{0}(\kappa)}, \quad \theta \in[-\pi, \pi)$,

where $I_{0}($.$) is the zero-order modified Bessel function, \theta_{p} \in[-\pi, \pi)$ accounts for the mean direction of AOA of scatter components, and $\kappa \geq 0$ controls the width of AOA of scatter components. Figs. 1 and 2 show $p_{\Theta}^{\text {scat }}(\theta)$ in linear and also in polar coordinates, respectively, for different values of $\kappa$ and $\theta_{p}=0$ (non-zero values of $\theta_{p}$ simply shift Fig. 1 circularly or rotate Fig. 2).

As can be observed in Fig. 1, for $\kappa=0$ we obtain $p_{\Theta}^{\text {scat }}(\theta)=1 / 2 \pi$ (isotropic scattering), while $\kappa=\infty$ yields $p_{\Theta}^{\text {scat }}(\theta)=\delta\left(\theta-\theta_{p}\right)$ (extremely non-isotropic scattering), where $\delta($.$) is the Dirac delta$ function. For small $\kappa$, this function approximates the cardioid PDF [21, p. 60], which is rather similar to the cosine PDF [20]; while for large $\kappa$ it resembles a Gaussian PDF with mean $\theta_{p}$ and standard deviation $1 / \sqrt{\kappa}[21$, p. 60]. In general, the von Mises PDF can approximate the wrapped Gaussian PDF quite well [21, p. 66]. It is interesting to note that the von Mises PDF appears in a number of other communication contexts. For example, this PDF is referred to as the Tikhonov PDF in partially coherent communication [22, p. 406], has been used in phase-locked loop related problems [23], and has been shown to represent the PDF of the phase of a sine wave in Gaussian noise for large signal to noise ratios [24].

A useful geometrical interpretation for $\kappa$ can be obtained by the fact that it is the slope of the tangent to the curve $p_{\Theta}^{\text {scat }}(\theta)$, in polar coordinates, at the point $\theta=\pi / 2$. This result can be obtained using the relation $\left(\rho \cos \theta+\rho^{\prime} \sin \theta\right) /\left(-\rho \sin \theta+\rho^{\prime} \cos \theta\right)$ [25, p. 176], where $\rho=\rho(\theta)$ represents a curve in polar coordinates and prime denotes differentiation with respect to $\theta$. A look at Fig. 2 verifies this result. For large $\kappa$, say $\kappa \geq 3, p_{\Theta}^{\text {scat }}(\theta)$ takes a unidirectional shape, as can be observed in Fig. 2. 
In order to obtain a measure for the spread of AOA's in the polar coordinates for large $\kappa$, we consider the inflexion points of $p_{\Theta}^{\text {scat }}(\theta)$ which are approximately equal to $\pm 1 / \sqrt{\kappa}$, assuming $\kappa$ is large [21, p.60]. So, for a unidirectional scattering scenario (large $\kappa$ ), the width of the AOA of scatter components at MS is roughly equal to $2 / \sqrt{\kappa}$. For $\kappa=3,10$, we obtain $66^{\circ}$ and $36^{\circ}$, respectively.

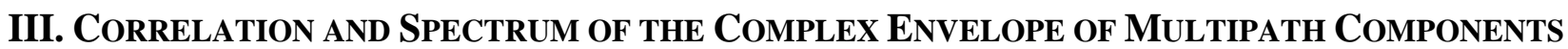

Let the received complex envelope at the MS due to scatter components be represented by $r^{\text {scat }}(t)=r_{i}^{\text {scat }}(t)+j r_{q}^{\text {scat }}(t)$, with $j=\sqrt{-1}$, where $r_{i}^{\text {scat }}(t)$ and $r_{q}^{\text {scat }}(t)$ are the in-phase and quadrature scatter components. Then, the normalized autocorrelation function of $r^{\text {scat }}(t), \tilde{\phi}_{r r}^{\text {scat }}(\tau)$, is given by $\tilde{\phi}_{r r}^{s c a t}(\tau)=\tilde{\phi}_{r_{i} r_{i}}^{s c a t}(\tau)+j \tilde{\phi}_{r_{i} r_{q}}^{s c a t}(\tau), \quad$ where $\quad \tilde{\phi}_{r_{i} r_{i}}^{\text {scat }}(\tau)=\tilde{\phi}_{r_{q} r_{q}}^{\text {scat }}(\tau) \quad$ and $\quad \tilde{\phi}_{r_{i} r_{q}}^{s c a t}(\tau)=-\tilde{\phi}_{r_{q} r_{i}}^{s c a t}(\tau) \quad$ are normalized autocorrelation and crosscorrelation functions of $r_{i}^{\text {scat }}(t)$ and $r_{q}^{\text {scat }}(t)$. Note that the term "normalized" indicates $\tilde{\phi}_{r r}^{s c a t}(0)=1$. It can be easily shown that $\tilde{\phi}_{r r}^{s c a t}(\tau)=E_{\Theta}\left[\exp \left(j 2 \pi f_{m} \tau \cos \Theta\right)\right][1, \mathrm{p}$. 40], where $E$ represents mathematical expectation and $f_{m}$ is the maximum Doppler frequency. Using $p_{\Theta}^{\text {scat }}(\theta)$ in $(1)$ for carrying out the expectation with respect to $\Theta$ yields [26, p. 357]:

$$
\tilde{\phi}_{r r}^{\text {scat }}(\tau)=\frac{I_{0}\left(\sqrt{\kappa^{2}-4 \pi^{2} f_{m}{ }^{2} \tau^{2}+j 4 \pi \kappa \cos \theta_{p} f_{m} \tau}\right)}{I_{0}(\kappa)} .
$$

For $\kappa=0,(2)$ results in the correlation functions for the Clarke's two-dimensional isotropic scattering model, i.e. $\tilde{\phi}_{r_{i} r_{i}}^{\text {scat }}(\tau)=I_{0}\left(j 2 \pi f_{m} \tau\right)=J_{0}\left(2 \pi f_{m} \tau\right)$ and $\tilde{\phi}_{r_{i} r_{q}}^{\text {scat }}(\tau)=0$, where $J_{0}($.$) is the zero-order Bessel$ function. Note that for both analytical and numerical calculations, formula (2) and its real and imaginary parts are much simpler than, and thus more preferable to the complicated results in [19].

The power spectrum of $r^{\text {scat }}(t)$ can be obtained based on $S_{r r}^{s c a t}(f)=\sigma^{2}\left[G(\theta) p_{\Theta}^{\text {scat }}(\theta)+G(-\theta) p_{\Theta}^{\text {scat }}(-\theta)\right] / \sqrt{f_{m}{ }^{2}-f^{2}},|f| \leq f_{m} \quad[1$, p.42], where $\theta$ and $f$ are related according to $\theta=\cos ^{-1}\left(f / f_{m}\right), \sigma^{2}$ is the total power from all scatter components at the input of the receiver antenna, and $G($.$) is the receiver antenna gain pattern. For a vertical monopole antenna,$ $G(\theta)=3 / 2$. So the normalized power spectrum of $r^{\text {scat }}(t)$ can be defined as $\tilde{S}_{r r}^{\text {scat }}(f)=2 S_{r r}^{\text {scat }}(f) / 3 \sigma^{2}$, i.e. $\quad \tilde{S}_{r r}^{\text {scat }}(f)=\left[p_{\Theta}^{\text {scat }}(\theta)+p_{\Theta}^{\text {scat }}(-\theta)\right] / \sqrt{f_{m}{ }^{2}-f^{2}}, \quad|f| \leq f_{m}$. The term "normalized" comes from $\int_{-f_{m}}^{f_{m}} \tilde{S}_{r r}^{\text {scat }}(f) d f=1$, which can be easily verified. This is in agreement with 
$\tilde{\phi}_{r r}^{s c a t}(0)=1$, since $\tilde{\phi}_{r r}^{s c a t}(\tau)$ and $\tilde{S}_{r r}^{s c a t}(f)$ are Fourier transform pairs. By using the $p_{\Theta}^{\text {scat }}(\theta)$ in $(1)$ and after some manipulations we obtain:

$$
\tilde{S}_{r r}^{s c a t}(f)=\frac{1}{\pi \sqrt{f_{m}{ }^{2}-f^{2}}} \frac{\exp \left(\kappa \cos \theta_{p} f / f_{m}\right) \cosh \left(\kappa \sin \theta_{p} \sqrt{1-\left(f / f_{m}\right)^{2}}\right)}{I_{0}(\kappa)}, \quad|f| \leq f_{m},
$$

where $\cosh ($.$) is the hyperbolic cosine. For \kappa=0$, (3) reduces to the power spectrum for the Clarke's two-dimensional isotropic scattering model, i.e. $\tilde{S}_{r r}^{\text {scat }}(f)=1 / \pi \sqrt{f_{m}{ }^{2}-f^{2}},|f| \leq f_{m}$.

For the specular component, the PDF of AOA can be written as:

$p_{\Theta}^{\text {spec }}(\theta)=\delta\left(\theta-\theta_{0}\right), \quad \theta \in[-\pi, \pi)$,

where $\theta_{0} \in[-\pi, \pi)$ is the AOA of the specular component. Let the received complex envelope at the MS due to the specular component be represented by $r^{\text {spec }}(t)$. Then, the normalized autocorrelation function of $r^{\text {spec }}(t)$ can be obtained based on $\tilde{\phi}_{r r}^{\text {spec }}(\tau)=E_{\Theta}\left[\exp \left(j 2 \pi f_{m} \tau \cos \Theta\right)\right]$ and using the given $p_{\Theta}^{\text {spec }}(\theta)$ :

$\tilde{\phi}_{r r}^{s p e c}(\tau)=\exp \left(j 2 \pi \cos \theta_{0} f_{m} \tau\right)$

The normalized power spectrum of $r^{s p e c}(t), \tilde{S}_{r r}^{s p e c}(f)$, can be easily obtained by taking the Fourier transform of (5):

$\tilde{S}_{r r}^{\text {spec }}(f)=\delta\left(f-f_{m} \cos \theta_{0}\right), \quad|f| \leq f_{m}$.

Note that $\tilde{\phi}_{r r}^{s p e c}(0)=\int_{-f_{m}}^{f_{m}} \tilde{S}_{r r}^{s p e c}(f) d f=1$.

When in addition to the scatter components, a specular component is also present, the PDF of the AOA of multipath components (scatter and specular) at the MS can be expressed as [19]:

$p_{\Theta}(\theta)=\frac{p_{\Theta}^{\text {scat }}(\theta)+K p_{\Theta}^{\text {spec }}(\theta)}{K+1}, \quad \theta \in[-\pi, \pi)$

where $K$ is the Rice factor, defined as the ratio of the power in the specular component to that in the scatter components. $p_{\Theta}^{\text {scat }}(\theta)$ and $p_{\Theta}^{\text {spec }}(\theta)$ are also given in (1) and (4), respectively. For $r(t)$, the received complex envelope at MS due to multipath components (scatter and specular), we also have 
$r(t)=\left[r^{\text {scat }}(t)+K r^{\text {spec }}(t)\right] / K+1$, where $r^{\text {scat }}(t)$ and $r^{\text {spec }}(t)$ are independent. Now, based on (7), the normalized autocorrelation function $\tilde{\phi}_{r r}(\tau)=E_{\Theta}\left[\exp \left(j 2 \pi f_{m} \tau \cos \Theta\right)\right]$ of $r(t)$ becomes:

$$
\tilde{\phi}_{r r}(\tau)=\frac{\tilde{\phi}_{r r}^{s c a t}(\tau)+K \tilde{\phi}_{r r}^{s p e c}(\tau)}{K+1},
$$

where $\tilde{\phi}_{r r}^{\text {scat }}(\tau)$ and $\tilde{\phi}_{r r}^{\text {spec }}(\tau)$ are given by (2) and (5), respectively. By defining $r_{i}(t)$ and $r_{q}(t)$ as the in-phase and quadrature multipath components of $r(t)$, i.e. $r(t)=r_{i}(t)+j r_{q}(t)$, we obtain $\tilde{\phi}_{r r}(\tau)=\tilde{\phi}_{r_{i} r_{i}}(\tau)+j \tilde{\phi}_{r_{i} r_{q}}(\tau)$, where $\quad \tilde{\phi}_{r_{i} r_{i}}(\tau)=\tilde{\phi}_{r_{q} r_{q}}(\tau) \quad$ and $\quad \tilde{\phi}_{r_{i} r_{q}}(\tau)=-\tilde{\phi}_{r_{q} r_{i}}(\tau) \quad$ are normalized autocorrelation and crosscorrelation functions of $r_{i}(t)$ and $r_{q}(t)$. The normalized power spectrum of $r(t)$ is simply the Fourier transform of $\tilde{\phi}_{r r}(\tau)$ in $(8)$, i.e.:

$\tilde{S}_{r r}(f)=\frac{\tilde{S}_{r r}^{s c a t}(f)+K \tilde{S}_{r r}^{s p e c}(f)}{K+1}$,

with $\tilde{S}_{r r}^{s c a t}(f)$ and $\tilde{S}_{r r}^{s p e c}(f)$ given by (3) and (6), respectively. For multipath components (scatter and specular) we have $\tilde{\phi}_{r r}(0)=\int_{-f_{m}}^{f_{m}} \tilde{S}_{r r}(f) d f=1$.

An important advantage of the PDF for AOA in (1) is the closed-from results obtained for $\tilde{\phi}_{r r}^{\text {scat }}(\tau)$ and $\tilde{S}_{r r}^{s c a t}(f)$, presented in (2) and (3), respectively. This can significantly simplify the derivation of correlation or spectral parameters, required for various system calculations. For example, assume the multipath components are such that $r^{\text {scat }}(t)$ is a zero-mean complex Gaussian process and $r^{\text {spec }}(t)=0$, hence $r(t)=r^{s c a t}(t)$ (this corresponds to the Rayleigh multipath fading channel, i.e. Rayleigh distribution for the envelope $\left|r^{\text {scat }}(t)\right|=\sqrt{\left[r_{i}^{\text {scat }}(t)\right]^{2}+\left[r_{q}^{\text {scat }}(t)\right]^{2}}$ and uniform distribution for the phase $\left.\angle r^{\text {scat }}(t)=\tan ^{-1}\left[r_{q}^{\text {scat }}(t) / r_{i}^{\text {scat }}(t)\right]\right)$. If we want to estimate the speed of the mobile [27], which is proportional to $f_{m}$, we may use the zero crossing rate of $r_{i}^{\text {scat }}(t)$, given by $Z C R\left\{r_{i}^{\text {scat }}(t)\right\}=\sqrt{\tilde{b}_{2}^{\text {scat }}} / \pi$ $[1, \mathrm{p} .60]$, where $\tilde{b}_{n}^{\text {scat }}$ is the normalized spectral moments of $r^{\text {scat }}(t)$, defined by:

$\tilde{b}_{n}^{s c a t}=(2 \pi)^{n} \int_{-f_{m}}^{f_{m}} f^{n} \tilde{S}_{r r}^{s c a t}(f) d f$.

Note that due to normalization, $\tilde{b}_{0}^{\text {scat }}=1$. Based on the Fourier relation between $\tilde{\phi}_{r r}^{\text {scat }}(\tau)$ and $\tilde{S}_{r r}^{\text {scat }}(f)$, we have $\tilde{b}_{n}^{\text {scat }}=j^{-n} d^{n} \tilde{\phi}_{r r}^{\text {scat }}(\tau) /\left.d \tau^{n}\right|_{\tau=0}$. Hence, according to (2), we derive a result more general than that given in [27]: 


$$
\tilde{b}_{2}^{s c a t}=\left(2 \pi f_{m}\right)^{2} \frac{\kappa\left[I_{0}(\kappa)+I_{2}(\kappa)\right] \cos ^{2} \theta_{p}+2 I_{1}(\kappa) \sin ^{2} \theta_{p}}{2 \kappa I_{0}(\kappa)} .
$$

So, for the zero crossing rate of $r_{i}^{\text {scat }}(t)$ we arrive at:

$$
Z C R\left\{r_{i}^{\text {scat }}(t)\right\}=\sqrt{\frac{\kappa\left[I_{0}(\kappa)+I_{2}(\kappa)\right] \cos ^{2} \theta_{p}+2 I_{1}(\kappa) \sin ^{2} \theta_{p}}{\kappa I_{0}(\kappa)}} \times \sqrt{2} f_{m} .
$$

The above closed-form formula is a useful tool for studying the effect of nonisotropic scattering on the performance of $Z C R\left\{r_{i}^{\text {scat }}(t)\right\}$ for estimating the speed of the mobile. For $\theta_{p}=0$ and $\kappa=0$, Clarke's isotropic scattering, the above result reduces to $\sqrt{2} f_{m}$, in agreement with [1, p. 60]; while for $\theta_{p}=\pi / 2$ and $\kappa=7$ we obtain $0.51 \times \sqrt{2} f_{m}$, half of the isotropic case. Therefore, we may conclude that $Z C R\left\{r_{i}^{\text {scat }}(t)\right\}$ is not a robust estimator against nonisotropic scattering and may change significantly as $\theta_{p}$ and $\kappa$ vary. Higher order spectral moments like $\tilde{b}_{3}^{\text {scat }}$ and $\tilde{b}_{4}^{\text {scat }}$, needed for developing more efficient and robust estimators for the speed [27], can be simply obtained by taking higher order derivatives of $\tilde{\phi}_{r r}^{\text {scat }}(\tau)$ in $(2)$.

\section{A Case Study}

Here we consider three different urban scenarios studied in [19] and derive the associated autocorrelation functions and power spectra of the complex envelope of multipath components at the MS. Let the MS move from left to right. For the first scenario, S1, scatter components arrive at the MS head on, i.e. $\theta_{p}=0$. For the second scenario, $\mathrm{S} 2$, scatter components arrive from both the $\theta_{p}=0$ and $\theta_{p}=\pi$ directions with equal probabilities. For the third scenario, S3, scatter components arrive at the MS from a direction perpendicular to its direction of motion, e.g. $\theta_{p}=\pi / 2$. For S1 and S3, $p_{\Theta}^{\text {scat }}(\theta)$ is equal to $\exp (\kappa \cos \theta) / 2 \pi I_{0}(\kappa)$ and $\exp (\kappa \sin \theta) / 2 \pi I_{0}(\kappa)$, respectively; while for $\mathrm{S} 2$ we have $\left.0.5 p_{\Theta}^{\text {scat }}(\theta)\right|_{\theta_{p}=0}+\left.0.5 p_{\Theta}^{\text {scat }}(\theta)\right|_{\theta_{p}=\pi}=\cosh (\kappa \cos \theta) / 2 \pi I_{0}(\kappa)$. These three PDF's are plotted in Fig. 3 for $\kappa=3$, together with Clarke's classic isotropic case $\kappa=0$.

The normalized autocorrelation functions of the complex envelope due to the scatter components for the above three scenarios can be derived according to (2): 


$$
\begin{aligned}
& \tilde{\phi}_{r r}^{\text {scat }, S 1}(\tau)=\frac{I_{0}\left(\sqrt{\kappa^{2}-4 \pi^{2} f_{m}{ }^{2} \tau^{2}+j 4 \pi \kappa f_{m} \tau}\right)}{I_{0}(\kappa)}, \\
& \tilde{\phi}_{r r}^{s c a t, S 2}(\tau)=\frac{\operatorname{Re}\left[I_{0}\left(\sqrt{\kappa^{2}-4 \pi^{2} f_{m}{ }^{2} \tau^{2}+j 4 \pi \kappa f_{m} \tau}\right)\right]}{I_{0}(\kappa)}, \\
& \tilde{\phi}_{r r}^{s c a t, S 3}(\tau)=\frac{I_{0}\left(\sqrt{\kappa^{2}-4 \pi^{2} f_{m}{ }^{2} \tau^{2}}\right)}{I_{0}(\kappa)},
\end{aligned}
$$

where $\mathrm{Re}[$.$] gives the real part of its complex argument. Notice that for S1, the imaginary part of$ $\tilde{\phi}_{r r}^{s c a t, S 1}(\tau)$, i.e. $\tilde{\phi}_{r_{i} r_{q}}^{\text {scat }, S 1}(\tau)$, is nonzero, contrary to Clarke's classic isotropic scenario. By substituting (10), (11), and (12) into (8), we can readily obtain $\tilde{\phi}_{r r}^{S 1}(\tau), \tilde{\phi}_{r r}^{S 2}(\tau)$, and $\tilde{\phi}_{r r}^{S 3}(\tau)$. Figs. 4 and 5 show $\tilde{\phi}_{r_{i} r_{i}}^{S 1}(\tau), \tilde{\phi}_{r_{i} r_{i}}^{S 2}(\tau)$, and $\tilde{\phi}_{r_{i} r_{i}}^{S 3}(\tau)$, the real parts of $\tilde{\phi}_{r r}^{S 1}(\tau), \tilde{\phi}_{r r}^{S 2}(\tau)$, and $\tilde{\phi}_{r r}^{S 3}(\tau)$, for $(K, \kappa)=(0,10)$ and $(1,3)$, respectively (for the case of nonzero $K$, we considered $\theta_{0}=\theta_{p}$ ). Comparing with Clarke's model $(K, \kappa)=(0,0)$ shown in Figs. 4 and 5, we observe the noticeable effect of non-isotropic scattering in different scenarios on the correlation properties at the MS. For nonisotropic scattering, it is possible to have large correlations when Clarke's isotropic scattering model predicts small correlations.

The normalized power spectra of the complex envelope due to the scatter components for the three scenarios $\mathrm{S} 1, \mathrm{~S} 2$, and $\mathrm{S} 3$ can be obtained based on (3):

$$
\begin{aligned}
& \tilde{S}_{r r}^{s c a t, S 1}(f)=\frac{1}{\pi \sqrt{f_{m}{ }^{2}-f^{2}}} \frac{\exp \left(\kappa f / f_{m}\right)}{I_{0}(\kappa)}, \quad|f| \leq f_{m}, \\
& \tilde{S}_{r r}^{s c a t, S 2}(f)=\frac{1}{\pi \sqrt{f_{m}{ }^{2}-f^{2}}} \frac{\cosh \left(\kappa f / f_{m}\right)}{I_{0}(\kappa)}, \quad|f| \leq f_{m}, \\
& \tilde{S}_{r r}^{s c a t, S 3}(f)=\frac{1}{\pi \sqrt{f_{m}{ }^{2}-f^{2}}} \frac{\cosh \left(\kappa \sqrt{1-\left(f / f_{m}\right)^{2}}\right)}{I_{0}(\kappa)},|f| \leq f_{m},
\end{aligned}
$$

Notice that for $\mathrm{S} 1$, the power spectrum $\tilde{S}_{r r}^{s c a t, S 1}(f)$ is not symmetric with respect to $f=0$, contrary to Clarke's scenario. By substituting (13), (14), and (15) into (9), we readily obtain $\tilde{S}_{r r}^{S 1}(f), \tilde{S}_{r r}^{S 2}(f)$, and 
$\tilde{S}_{r r}^{S 3}(f)$. Figs. 6 and 7 show $f_{m} \tilde{S}_{r r}^{s c a t, S 1}(f), f_{m} \tilde{S}_{r r}^{s c a t, S 2}(f)$, and $f_{m} \tilde{S}_{r r}^{s c a t, S 3}(f)$ for $\kappa=10$ and 3 , respectively. Comparing with Clarke's classic isotropic case, $\kappa=0$, superimposed on the plots in Figs. 6 and 7, we clearly see the significant effects of different non-isotropic scattering scenarios on the power spectrum at the MS.

\section{EXPERIMENTAL RESULTS}

In this section we show the utility of the proposed AOA distribution, and the associated parametric correlation function in describing the correlation properties of a range of a variety of measured data. First we give a brief summary of the data used (the dynamic statistical characteristics of this data set are discussed in [28]). In the subsequent subsections, we talk about envelope correlation and envelope extraction, two non-isotropic scattering correlation model for fitting to data, estimation of the envelope correlation of data and parameter estimation for the two models, and finally, the merits and limitations of the two models in fitting to measured data.

\section{A. Test Locations and Data Collection}

Two sites were used for the collection of data: a suburban housing development in Greenville, Texas, and an urban area among the buildings of the campus of the University of Texas at Arlington, Texas. These test locations were chosen to provide a random signal environment that would be representative of actual operational situations. By choosing environments with irregular structures, data reflects the general angular spread of the signal and not yield results found only in very regular environments.

In the suburban area, parked vehicles, vegetation, and inconsistency in the setback of the housing structures produced very irregular reflective conditions. Measurements for records \#0011 through \#0015 were taken in the suburban neighborhood characterized by homes of a wood/brick construction with a nominal $7 \mathrm{~m}$ of separation between the structures. The transmit antenna was at a height of $2 \mathrm{~m}$ above a hillside location to produce a low grazing angle over the homes, simulating a cellular radio signal at a greater distance. The measurements were made with the receive antenna in a total shadow 
environment. Records \#0016 through \#0018 represent data taken near a large intersection in a suburban area with the transmit antenna height raised to about $5 \mathrm{~m}$. For the intersection measurements there was a significant direct ray component of the signal. During these sample periods there was significant traffic activity near the test vehicle.

The urban environment is characterized by buildings of one to six stories in height, heavy traffic, large open areas, and highly inconsistent setback from the street. Records \#0019 through \#0022 were made in the urban location with the transmit antenna at a height of about $21 \mathrm{~m}$, producing a signal at a moderately low angle of radiation over the buildings surrounding the path. A map of the urban location is shown in Fig. 8. Buildings shown are of modern brick construction. Building heights are indicated by a number inside the outlines shown in Fig. 8. The urban location contained significant vehicular traffic, and the measurements were made over paths that had no direct ray component, but demonstrated the deep shadow environment.

The UHF transmitter generated a $910.25 \mathrm{MHz}$ carrier at a nominal power of $0.2 \mathrm{~W}$ (the wavelength was $0.33 \mathrm{~m}$ ). Signal samples were taken at distances of $0.16 \mathrm{~km}$ to $0.25 \mathrm{~km}$. The collected data consist of twelve sets of narrowband inphase and quadrature components taken at twelve different locations. Each data set represents the signal over a traveled distance of $47 \mathrm{~m}$, or $7 \mathrm{~s}$ of time. These signals were digitized with a sampling rate of $35156.25 \mathrm{~Hz}$, to produce one sample per $0.2 \mathrm{~mm}$ of distance traveled ( $28 \mu \mathrm{s}$ in time) by the moving receiver platform (at a fixed speed of $6.7 \mathrm{~m} / \mathrm{s}$ ). Hence, the number of samples for each record is approximately 250,000. The antenna at the mobile receiver was a $1 / 4$ wave vertical stub, $0.6 \mathrm{~cm}$ in diameter, mounted on top of a $1.9 \mathrm{~cm}$ diameter aluminum tube. The tube served as a rather small ground plane. For the fixed transmitter, the same antenna was used except a 1/4 wave diameter ground plane (flat) was attached to the base of the antenna stub. Both antennas had omnidirectional patterns.

\section{B. Envelope Correlation and Envelope Extraction}

Since for many practical applications, the envelope of multipath components and its correlation properties are of concern, we consider the envelope of multipath component $z(t)=|r(t)|$. When $r(t)$ is 
a complex Gaussian process with non-zero mean, the autocovariance function of $z^{2}(t)$, $\mu_{z^{2} z^{2}}(\tau)=E\left[\left\{z^{2}(t)-E\left[z^{2}(t)\right]\right\}\left\{z^{2}(t+\tau)-E\left[z^{2}(t+\tau)\right]\right\}\right], \quad$ can be expressed in terms of the autocorrelation function of the complex envelope of multipath components $r(t)$, having both scatter and specular components [29]. The normalized version of $\mu_{z^{2} z^{2}}(\tau), \tilde{\mu}_{z^{2} z^{2}}(\tau)$, assuming no specular component (which makes $r(t)$ a zero-mean complex Gaussian process), is given by [1, p. 55]:

$\tilde{\mu}_{z^{2} z^{2}}^{s c a t}(\tau)=\left|\tilde{\phi}_{r r}^{s c a t}(\tau)\right|^{2}$,

where $\tilde{\mu}_{z^{2} z^{2}}^{\text {scat }}(\tau)$ is the normalized autocovariance function of $z^{2}(t)$ due to scatter components. Again "normalized" indicates $\tilde{\mu}_{z^{2} z^{2}}^{\text {scat }}(0)=1$.

In order to extract $z(t)$, the multipath (or fast fading) component of the envelope, which we are interested in, the shadow (or slow fading) component of the envelope must be removed. Let $R(t)=R_{i}(t)+j R_{q}(t)$ denote the received complex envelope at the MS in the presence of both multipath and shadow components, with $R_{i}(t)$ and $R_{q}(t)$ as the inphase and the quadrature components. The shadow (or slow fading) component of the envelope, $|R(t)|$, is represented by its mean, $E[|R(t)|]$, which is a function of time because $|R(t)|$ is nonstationary. The multipath (or fast fading) component of $|R(t)|$ can be represented as $z(t)=|R(t)| / E[|R(t)|][1$, p. 91]. A method for estimating $E[|R(t)|]$ from $|R(t)|$ using a local sliding window is described in [30].

\section{Two Non-Isotropic Scattering Propagation Model}

We consider $\tilde{\mu}_{z^{2} z^{2}}^{\text {scat }}(\tau)$ for fitting to the data, according to two propagation models: the simple nonisotropic model and the composite non-isotropic/isotropic model. In the simple non-isotropic model, we assume that the MS receives the signals mainly from one direction. The AOA PDF and the normalized autocorrelation function for the simple non-isotropic model are given in (1) and (2), respectively. For the composite non-isotropic/isotropic model, the MS receives signals uniformly from all directions, in addition to a component centered around a specific direction. The AOA PDF and the normalized autocorrelation function for the composite non-isotropic/isotropic model can be written as: 


$$
\begin{aligned}
& p_{\Theta}^{\text {scat }}(\theta)=\zeta \frac{\exp \left[\kappa \cos \left(\theta-\theta_{p}\right)\right]}{2 \pi I_{0}(\kappa)}+(1-\zeta) \frac{1}{2 \pi}, \quad \theta \in[-\pi, \pi), \\
& \tilde{\phi}_{r r}^{\text {scat }}(\tau)=\zeta \frac{I_{0}\left(\sqrt{\kappa^{2}-4 \pi^{2} f_{m}^{2} \tau^{2}+j 4 \pi \kappa \cos \theta_{p} f_{m} \tau}\right)}{I_{0}(\kappa)}+(1-\zeta) J_{0}\left(2 \pi f_{m} \tau\right),
\end{aligned}
$$

where $0 \leq \zeta \leq 1$ indicates the amount of directional reception. The composite non-isotropic/isotropic model reduces to the simple non-isotropic model for $\zeta=1$, and simplifies to Clarke's model for $\zeta=0$. For the simple non-isotropic model and based on (2) and (16) we have:

$$
\tilde{\mu}_{z^{2} z^{2}}^{\text {scat }}(\tau)=\frac{\left|I_{0}\left(\sqrt{\kappa^{2}-4 \pi^{2} f_{m}^{2} \tau^{2}+j 4 \pi \kappa \cos \theta_{p} f_{m} \tau}\right)\right|^{2}}{I_{0}{ }^{2}(\kappa)}
$$

while for the composite non-isotropic/isotropic model and according to (18) and (16) we derive:

$$
\tilde{\mu}_{z^{2} z^{2}}^{\text {scat }}(\tau)=\frac{\left|\zeta I_{0}\left(\sqrt{\kappa^{2}-4 \pi^{2} f_{m}^{2} \tau^{2}+j 4 \pi \kappa \cos \theta_{p} f_{m} \tau}\right)+(1-\zeta) J_{0}\left(2 \pi f_{m} \tau\right) I_{0}(\kappa)\right|^{2}}{I_{0}^{2}(\kappa)} .
$$

\section{Estimation of the Envelope Correlation and the Parameters of the Two Models}

The estimate for the normalized autocovariance function of the envelope-squared due to multipath components, $\hat{\tilde{\mu}}_{z^{2} z^{2}}(\tau)$, was obtained using the biased method [31, p. 743], scaled such that $\hat{\tilde{\mu}}_{z^{2} z^{2}}(0)=1$ (the bias is very small because of the large number of samples 250000). Recall that (16) and consequently (19) and (20) are valid when $r(t)$ is a zero-mean complex Gaussian process (no specular component hence $r^{\text {spec }}(t)=0$ and $\left.r(t)=r^{\text {scat }}(t)\right)$. This means that at least the univariate PDF's of the envelope, $z(t)=|r(t)|$, and the phase, $\angle r(t)$, have to be Rayleigh and uniform, respectively, independent of each other (note that a univariate Rayleigh PDF for the envelope by itself does not imply that $r(t)$ is a zero-mean complex Gaussian process [32]). According to the data analysis results partly reported in [33], records \#0011, \#0017, \#0019, \#0021, and \#0022 meet the above requirements. So, for these records, equation (16), and subsequently (19) and (20) hold exactly, while for other records, the three equations may be considered as approximations. However, our collected data that is 
analyzed and discussed in the sequel, suggest that irrespective of the apparent underlying probability distribution for the complex envelope $r(t)$, the parametric models given in (19) and (20) give very good fits to the autocovariance function of the envelope-squared.

In (19) and (20), the maximum Doppler frequency is $f_{m}=20 \mathrm{~Hz}$. The unknown parameters $\left(\kappa, \theta_{p}\right)$ in (19) for the simple non-isotropic model and $\left(\kappa, \theta_{p}, \zeta\right)$ in $(20)$ for the composite nonisotropic/isotropic model were estimated by the nonlinear least squares method (which provides consistent estimates $\quad[34, \quad$ p. $\quad 207]): \quad\left(\hat{\kappa}, \hat{\theta}_{p}\right)=\underset{\left(\kappa, \theta_{p}\right)}{\arg \min \mathrm{MSE}_{\text {Simple }}} \quad$ where $\operatorname{MSE}_{\text {Simple }}=n^{-1} \sum_{\ell=1}^{n}\left[\hat{\tilde{\mu}}_{z^{2} z^{2}}\left(\tau_{\ell}\right)-\tilde{\mu}_{z^{2} z^{2}}^{\text {scat }}\left(\tau_{\ell}\right)\right]^{2} \quad$ with $\quad \tilde{\mu}_{z^{2} z^{2}}^{\text {scat }}(\tau) \quad$ given $\quad$ in $\quad$ (19), while $\left(\hat{\kappa}, \hat{\theta}_{p}, \hat{\zeta}\right)=\underset{\left(\kappa, \theta_{p}, \zeta\right)}{\arg \min } \mathrm{MSE}_{\text {Composite }}$ where $\operatorname{MSE}_{\text {Composite }}=n^{-1} \sum_{\ell=1}^{n}\left[\hat{\tilde{\mu}}_{z^{2} z^{2}}\left(\tau_{\ell}\right)-\tilde{\mu}_{z^{2} z^{2}}^{\text {scat }}\left(\tau_{\ell}\right)\right]^{2}$ with $\tilde{\mu}_{z^{2} z^{2}}^{\text {scat }}(\tau)$ given in (20) (note that MSE stands for mean-squared-error). In the estimation procedure we had $n=350$ and $\tau_{\ell+1}-\tau_{\ell}=0.14 \times 10^{-3} \mathrm{~s}, \ell=1, \ldots, n-1$. This indicates that the estimation was carried out over the coherence time [1, p. 72], which ranges from $\tau=0$ through $\tau=1 / f_{m}$. The estimated values $\left(\hat{\kappa}, \hat{\theta}_{p}\right)$ and $\left(\hat{\kappa}, \hat{\theta}_{p}, \hat{\zeta}\right)$ for both models are listed in Table I. The mean-squared-error of the Clarke's model, defined by $\mathrm{MSE}_{\text {Clarke }}=n^{-1} \sum_{\ell=1}^{n}\left[\hat{\tilde{\mu}}_{z^{2} z^{2}}\left(\tau_{\ell}\right)-J_{0}{ }^{2}\left(2 \pi f_{m} \tau_{\ell}\right)\right]^{2}$, is also given in Table I, together with the minimum values of $\mathrm{MSE}_{\text {Simple }}$ and $\mathrm{MSE}_{\text {Composite }}$ for all twelve data sets. In Fig. 9 and for several data records, $\tilde{\mu}_{z^{2} z^{2}}^{s c t}(\tau)$ 's in (19) and (20) are plotted using the estimated parameters $\left(\hat{\kappa}, \hat{\theta}_{p}\right)$ and $\left(\hat{\kappa}, \hat{\theta}_{p}, \hat{\zeta}\right)$, together with $\hat{\tilde{\mu}}_{z^{2} z^{2}}(\tau)$ and the associated Clarke's result $J_{0}{ }^{2}\left(2 \pi f_{m} \tau\right)$. The correlation plots of records \#0012, \#0020, \#0021, and also \#0015 are not shown in Fig. 9, as the first three are similar to the correlation plot of \#0011, whereas the forth one resembles that of \#0014.

\section{E. Discussion}

As expected, $\mathrm{MSE}_{\text {Clarke }}>\min \left(\mathrm{MSE}_{\text {Simple }}\right) \geq \min \left(\mathrm{MSE}_{\text {Composite }}\right)$ for all the twelve sets of data. Note the significant error of Clarke's isotropic scattering model in predicting the amount of correlation for most of the cases. For example, for records $\# 0011$ and $\# 0014$, the maximum error in predicting the correlations is about 0.5 , which is not negligible at all. Regarding the characteristics of the two proposed models, let us first focus on the simple non-isotropic model. In this case, only for few situations the maximum deviation of the model predictions from the empirical correlations over the 
range $0<f_{m} \tau<1$ is about 0.2 , and for the rest it is less than 0.1 . In general, for $\hat{\kappa}>1$, the empirical correlations approach zero slower than the predictions of Clarke's model. Notice the very slow convergence of the empirical correlations to zero for record $\# 0018, \hat{\kappa}=3.3$, followed well by the simple non-isotropic model. Only for one case (record \#0019), $\hat{\kappa}$ is small and equal to 0.6, yielding a result close to that given by Clarke's model. One disadvantage of the simple non-isotropic model, which gave us the motivation to develop the composite non-isotropic/isotropic model, is its inability to follow the oscillations observed in the empirical curves of records \#0013 - \#0017 and \#0022. On the other hand, the composite non-isotropic/isotropic model is able to follow all kinds of variations of the empirical curves. Specifically, note the improvements obtained in data fitting for records \#0013 $\# 0017, \# 0019$, and \#0022. Of course those improvements are achieved at the expense of adding the parameter $\zeta$ to the simple non-isotropic model.

Note that since the simple non-isotropic model should account for the incoming waves at the MS from all directions just by a single von Mises PDF, estimated values $\hat{\kappa}$ are all small for this model ( $\hat{\kappa} \leq 3.3)$, which correspond to large AOA spreads $\left(2 / \sqrt{\hat{\kappa}} \geq 63^{\circ}\right)$. On the other hand, since the composite non-isotropic/isotropic model is equipped with two PDF for modeling the directions of incoming waves at the MS, von Mises PDF can take care of the directional reception much better, which in turn yields moderate and large values for $\hat{\kappa}(12 \leq \hat{\kappa} \leq 88$ and $\hat{\kappa}=700)$. This corresponds to moderate and small values for the AOA spread of the directional incoming waves at the MS $\left(12^{\circ} \leq 2 / \sqrt{\hat{\kappa}} \leq 33^{\circ}\right.$ and $\left.2 / \sqrt{\hat{\kappa}}=4.3^{\circ}\right)$.

Regarding the physical interpretation of the estimated values $\hat{\theta}_{p}$ for both models is Table I, let us assume that a $\pm 20^{\circ}$ tolerance in estimating the approximate orientations to the fixed transmitter, listed in Table I, is acceptable (of course the tolerance range $\pm 20^{\circ}$ should depend on $\hat{\kappa}$, but here we fix it to make a simple conclusion). In Table I, a "perpendicular" direction corresponds to either $\theta_{p}=90^{\circ}$ or $\theta_{p}=270^{\circ}$, and a "parallel" direction is associated with either $\theta_{p}=0^{\circ}$ or $\theta_{p}=180^{\circ}$. Now it is easy to verify that within the tolerance limit $\pm 20^{\circ}$, the simple non-isotropic model correctly estimates $\theta_{p}$ only for records $\# 0013, \# 0014, \# 0018$, and $\# 0021$, while the composite non-isotropic/isotropic model successfully estimates $\theta_{p}$ for \#0011 - \#0013, \#0016, \#0017, \#0020 and \#0022. This confirms the 
superiority of the composite non-isotropic/isotropic model in characterizing the propagation of waves in wireless channels.

\section{ConClusion}

In this contribution we have proposed the application of the von Mises PDF for modeling the distribution of the angles of arrival of scatter components received at the mobile station. This flexible parametric family of angular PDF's includes a variety of non-isotropic scattering scenarios, including the isotropic one as a special case. This PDF for angles of arrival results in closed-form and easy-touse formulas for the correlation function and power spectrum of the complex envelope of scatter components at the mobile station. These formulas include Clarke's Bessel function-based correlation and U-shaped power spectrum as special cases. As a consequence, those correlation or spectral parameters which are important for various system calculations can be easily derived. For example, spectral moments of the received signal at the mobile station, useful for estimating the speed of mobile, can be expressed in simple closed forms. The mathematical tractability of the correlation function and power spectrum derived in this paper make them useful for other applications such as efficient simulation of fading channels [36], Karhunen-Loeve expansion of the multipath fading process [37], derivation of closed-form expressions for the error probabilities in channels with significant Doppler spread, hence having fast variations [38] [39, pp. 523-524] [40, pp. 365-367] (in place of numericalonly calculations [41]), etc.. The utility of the proposed model and new results have been verified by fitting the parametric model of the correlation function to the estimates of this function based on measured data.

\section{REFERENCES}

[1] G. L. Stuber, Principles of Mobile Communication. Boston, MA: Kluwer, 1996.

[2] J. S. Sadowsky and V. Kafedziski, "On the correlation and scattering functions of the WSSUS channel for mobile communication," IEEE Trans. Vehic. Technol., vol. 47, pp. 270-282, 1998. 
[3] M. Patzold, Y. Li, and F. Laue, "A study of a land mobile satellite channel model with asymmetrical Doppler power spectrum and lognormally distributed line-of-sight component," IEEE Trans. Vehic. Technol., vol. 47, pp. 297-310, 1998.

[4] M. Patzold, U. Killat, Y. Li, and F. Laue, "Modeling, analysis, and simulation of nonfrequencyselective mobile radio channels with asymmetrical Doppler power spectral density shapes," IEEE Trans. Vehic. Technol., vol. 46, pp. 494-507, 1997.

[5] F. P. Fontan, M. A. V. Castro, J. Kunisch, J. Pamp, E. Zollinger, S. Buonomo, P. Baptista, and B. Arbesser, "A versatile framework for a narrow-and wide-band statistical propagation model for the LMS channel," IEEE Trans. Broadcasting, vol. 43, pp. 431-458, 1997.

[6] W. R. Braun and U. Dersch, "A physical mobile radio channel model," IEEE Trans. Vehic. Technol., vol. 40, pp. 472-482, 1991.

[7] A. Kuchar, E. A. Aparicio, J. P. Rossi, and E. Bonek, "Azimuth, elevation, and delay of signals at mobile station site," in Wireless Personal Communications: Emerging Technologies for Enhanced Communications. W. H. Tranter, T. S. Rappaport, B. D. Woerner, and J. H. Reed, Eds., Boston, MA: Kluwer, 1999, pp. 99-110.

[8] J. P. Rossi, J. P. Barbot, and A. J. Levy, "Theory and measurement of the angle of arrival and time delay of UHF radio waves using a ring array," IEEE Trans. Antennas Propagat., vol. 45, pp. 876-884, 1997.

[9] J. Fuhl, J. P. Rossi, and E. Bonek, "High-resolution 3-D direction-of-arrival determination for urban mobile radio,” IEEE Trans. Antennas Propagat., vol. 45, pp. 672-682, 1997.

[10] Q. Spencer, M. Rice, B. Jeffs, and M. Jensen, “A statistical model for angle of arrival in indoor multipath propagation," in Proc. IEEE Vehic. Technol. Conf., Phoenix, AZ, 1997, pp. 14151419.

[11] P. C. Fannin and A. Molina, "Analysis of mobile radio channel sounding measurements in inner city Dublin at 1.808 Ghz,” IEE Proc. Commun., vol. 143, pp. 311-316, 1996.

[12] J. G. Wang, A. S. Mohan, and T. A. Aubrey, “Angles-of-arrival of multipath signals in indoor environments," in Proc. IEEE Vehic. Technol. Conf., Atlanta, GA, 1996, pp. 155-159. 
[13] S. Guerin, "Indoor wideband and narrowband propagation measurements around $60.5 \mathrm{GHz}$ in an empty and furnished room," in Proc. IEEE Vehic. Technol. Conf., Atlanta, GA, 1996, pp. 160164.

[14] T. Lo and J. Litva, "Angles of arrival of indoor multipath," Electron. Lett., vol. 28, pp. 16871689, 1992.

[15] D. O. Reudink, "Large-scale variations of the average signal," in Microwave Mobile Communications. W. C. Jakes, Jr., Ed., New York: Wiley, 1974, pp. 79-131.

[16] W. C. Y. Lee, "Finding the approximate angular probability density function of wave arrival by using a directional antenna," IEEE Trans. Antennas Propagat., vol. 21, pp. 328-334, 1973.

[17] P. Petrus, J. H. Reed, and T. S. Rappaport, "Effects of directional antennas at the base station on the Doppler spectrum," IEEE Commun. Lett., vol. 1, pp. 40-42, 1997.

[18] J. C. Liberti and T. S. Rappaport, "A geometrically based model for line-of-sight multipath radio channels," in Proc. IEEE Vehic. Technol. Conf., Atlanta, GA, 1996, pp. 844-848.

[19] K. Anim-Appiah, "Complex envelope correlations for non-isotropic scattering," Electron. Lett., vol. 34, pp. 918-919, 1998.

[20] M. D. Austin and G. L. Stuber, "Velocity adaptive handoff algorithms for microcellular systems," IEEE Trans. Vehic. Technol., vol. 43, pp. 549-561, 1994.

[21] K. V. Mardia, Statistics of Directional Data. London: Academic, 1972.

[22] M. K. Simon, S. M. Hinedi, and W. C. Lindsey, Digital Communication Techniques- Signal Design and Detection. Upper Saddle River, NJ: PTR Prentice Hall, 1995.

[23] A. J. Viterbi, "Phase-locked loop dynamics in the presence of noise by Fokker-Planck techniques," Proc. IEEE, vol. 51, pp. 1737-1753, 1963.

[24] H. Leib and S. Pasupathy, "The phase of a vector perturbed by Gaussian noise and differentially coherent receivers", IEEE Trans. Inform. Theory, vol. 34, pp. 1491-1501, 1988.

[25] F. Ayres, Jr. and E. Mendelson, Theory and Problems of Differential and Integral Calculus, 3rd ed., New York: McGraw-Hill, 1990.

[26] I. S. Gradshteyn and I. M. Ryzhik, Table of Integrals, Series, and Products, 5th ed., A. Jeffrey, 
Ed., San Diego, CA: Academic, 1994.

[27] A. Abdi and M. Kaveh, "A new velocity estimator for cellular systems based on higher order crossings," in Proc. Asilomar Conf. Signals, Systems, Computers, Pacific Grove, CA, 1998, pp. 1423-1427.

[28] H. Allen Barger, "Dynamic characteristics of a narrowband land mobile communication channel," IEEE Trans. Vehic. Technol., vol. 47, pp. 216-224, 1998.

[29] G. L. Stuber, private communication, 1999.

[30] A. Abdi and M. Kaveh, "On the utility of the gamma PDF in modeling shadow fading (slow fading)," in Proc. IEEE Vehic. Technol. Conf., Houston, TX, 1999, pp. 2308-2312.

[31] A. V. Oppenheim and R. W. Schafer, Discrete-Time Signal Processing. Englewood Cliffs, NJ: Prentice Hall, 1989.

[32] S. H. Lin, "Statistical behavior of a fading signal," Bell Syst. Tech. J., vol. 50, pp. 3211-3270, 1971.

[33] A. Abdi, H. A. Barger, and M. Kaveh, "Signal modeling in wireless fading channels using spherically invariant processes," in Proc. IEEE Int. Conf. Acoust., Speech, Signal Processing, Istanbul, Turkey, 2000, pp. 2997-3000.

[34] R. Gonin and A. H. Money, Nonlinear $L_{p}$-Norm Estimation. New York: Marcel Dekker, 1989.

[35] R. Von Mises, "Über die "Ganzzahligkeit” der Atomgewicht und verwandte Fragen," Physikal. Z., vol. 19, pp. 490-500, 1918.

[36] K. W. Yip and T. S. Ng, "Karhunen-Loeve expansion of the WSSUS channel output and its application to efficient simulation," IEEE J. Select. Areas Commun., vol. 15, pp. 640-646, 1997.

[37] M. Visintin, "Karhunen-Loeve expansion of a fast Rayleigh fading process," Electron. Lett., vol. 32, pp. 1712-1713, 1996.

[38] P. A. Bello and B. D. Nelin, "The influence of fading spectrum on the binary error probabilities of incoherent and differentially coherent matched filter receivers," IRE Trans. Commun. Syst., vol. 10, pp. 160-168, 1962.

[39] W. C. Jakes, Jr., Ed., Microwave Mobile Communications. New York: Wiley, 1974. 
[40] M. D. Yacoub, Foundations of Mobile Radio Engineering. Boca Raton, FL: CRC, 1993.

[41] T. B. Welch, M. J. Walker, and R. E. Ziemer, "Effects of directional antennas with realizable beam patterns on the spaced-time correlation function," in Wireless Personal Communications: Emerging Technologies for Enhanced Communications. W. H. Tranter, T. S. Rappaport, B. D. Woerner, and J. H. Reed, Eds., Boston, MA: Kluwer, 1999, pp. 1-9. 


\section{TABLE I}

ESTIMATED VALUES FOR THE PARAMETERS OF THE SIMPLE NON-ISOTROPIC AND COMPOSITE NONISOTROPIC/ISOTROPIC MODELS, TOGETHER WITH THE ESTIMATION ERRORS

\begin{tabular}{|c|c|c|c|c|c|c|c|c|c|}
\hline \multirow[b]{2}{*}{$\begin{array}{l}\text { Record } \\
\text { no. }\end{array}$} & \multirow{2}{*}{$\begin{array}{l}\text { Approximate } \\
\text { orientation to } \\
\text { transmitter }\end{array}$} & \multirow{2}{*}{$\begin{array}{c}\begin{array}{c}\text { Clarke's } \\
\text { model }\end{array} \\
\text { MSE }_{\text {Clarke }}\end{array}$} & \multicolumn{3}{|c|}{$\begin{array}{l}\text { The simple non-isotropic } \\
\text { model }\end{array}$} & \multicolumn{4}{|c|}{$\begin{array}{l}\text { The composite non-isotropic/isotropic } \\
\text { model }\end{array}$} \\
\hline & & & $\hat{\kappa}$ & $\hat{\theta}_{p}$ & $\min \left(\mathrm{MSE}_{\text {Simple }}\right)$ & $\hat{\kappa}$ & $\hat{\theta}_{p}$ & $\hat{\zeta}$ & $\min \left(\mathrm{MSE}_{\text {Composite }}\right)$ \\
\hline 0011 & Perpendicular & $1.2 \mathrm{E}-1$ & 2.4 & $19.8^{\circ}$ & $3.3 \mathrm{E}-4$ & 38 & $280.8^{\circ}$ & 0.80 & $3.3 \mathrm{E}-4$ \\
\hline 0012 & Perpendicular & $9.3 \mathrm{E}-2$ & 3.0 & $36.0^{\circ}$ & $1.1 \mathrm{E}-3$ & 29 & $266.4^{\circ}$ & 0.78 & $2.5 \mathrm{E}-4$ \\
\hline 0013 & Parallel & $4.6 \mathrm{E}-2$ & 1.2 & $0^{\circ}$ & $1.5 \mathrm{E}-2$ & 700 & $0^{\circ}$ & 0.50 & $3.0 \mathrm{E}-3$ \\
\hline 0014 & Parallel & $1.6 \mathrm{E}-1$ & 2.4 & $0^{\circ}$ & $7.0 \mathrm{E}-3$ & 12 & $331.2^{\circ}$ & 0.80 & $2.9 \mathrm{E}-4$ \\
\hline 0015 & Perpendicular & $1.6 \mathrm{E}-1$ & 2.4 & $0^{\circ}$ & $1.7 \mathrm{E}-2$ & 36 & $331.2^{\circ}$ & 0.72 & $9.7 \mathrm{E}-4$ \\
\hline 0016 & Perpendicular & $2.3 \mathrm{E}-2$ & 1.2 & $36.0^{\circ}$ & $9.8 \mathrm{E}-3$ & 700 & $108.0^{\circ}$ & 0.42 & $3.1 \mathrm{E}-3$ \\
\hline 0017 & Perpendicular & $5.2 \mathrm{E}-2$ & 1.5 & $19.8^{\circ}$ & $8.9 \mathrm{E}-3$ & 700 & $79.2^{\circ}$ & 0.56 & $4.1 \mathrm{E}-4$ \\
\hline 0018 & Parallel & $2.5 \mathrm{E}-1$ & 3.3 & $0^{\circ}$ & $3.4 \mathrm{E}-3$ & 88 & $252.0^{\circ}$ & 0.86 & $7.8 \mathrm{E}-4$ \\
\hline 0019 & Perpendicular & $6.7 \mathrm{E}-3$ & 0.6 & $0^{\circ}$ & $2.7 \mathrm{E}-3$ & 700 & $237.6^{\circ}$ & 0.30 & $7.6 \mathrm{E}-4$ \\
\hline 0020 & Perpendicular & $1.1 \mathrm{E}-1$ & 2.1 & $14.4^{\circ}$ & $1.5 \mathrm{E}-3$ & 59 & $79.20^{\circ}$ & 0.74 & $1.5 \mathrm{E}-4$ \\
\hline 0021 & Parallel & $1.2 \mathrm{E}-1$ & 2.1 & $10.8^{\circ}$ & $2.0 \mathrm{E}-4$ & 39 & $259.2^{\circ}$ & 0.80 & $1.8 \mathrm{E}-4$ \\
\hline 0022 & Perpendicular & $6.9 \mathrm{E}-2$ & 1.5 & $0^{\circ}$ & $1.1 \mathrm{E}-2$ & 700 & $252.0^{\circ}$ & 0.60 & $1.3 \mathrm{E}-3$ \\
\hline
\end{tabular}




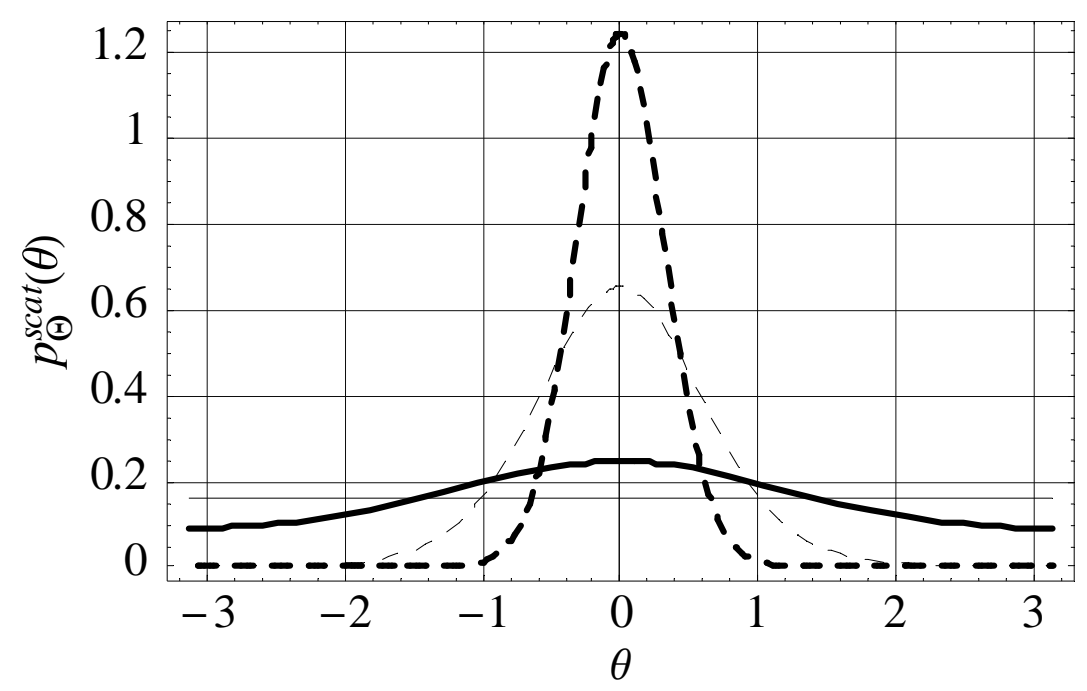

Fig. 1. Von Mises PDF for the angle of arrival of scatter components at the mobile station $\left(\theta_{p}=0\right)$.
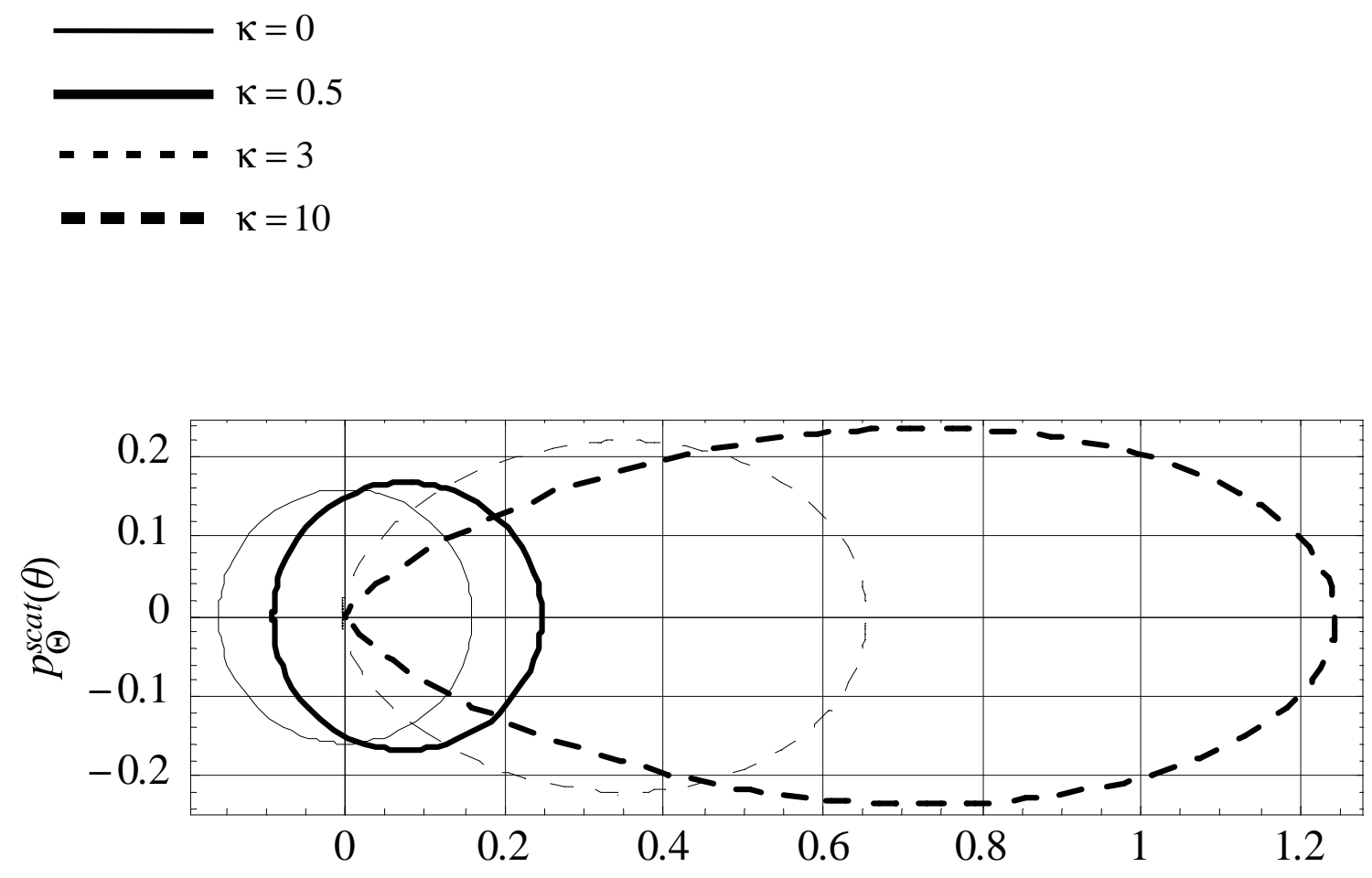

Fig. 2. Von Mises PDF in polar coordinates for the angle of arrival of scatter components at the mobile station $\left(\theta_{p}=0\right)$.

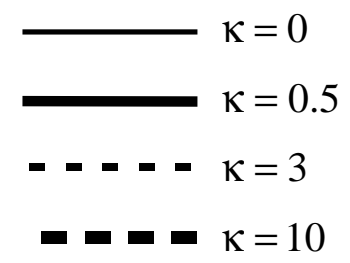




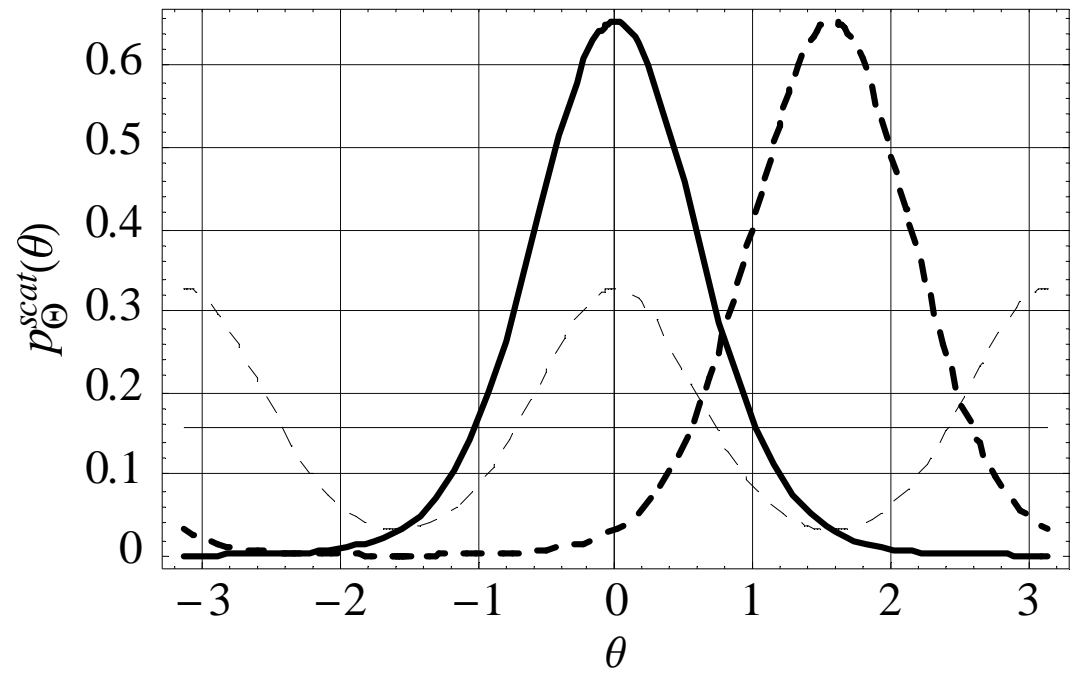

Fig. 3. The PDF of angle of arrival of scatter components at the mobile station for different urban scenarios.

Clarke's scenario: $\kappa=0$

S1 scenario: $\kappa=3$

- - - - S2 scenario: $\kappa=3$

- - - - S3 scenario: $\kappa=3$ 


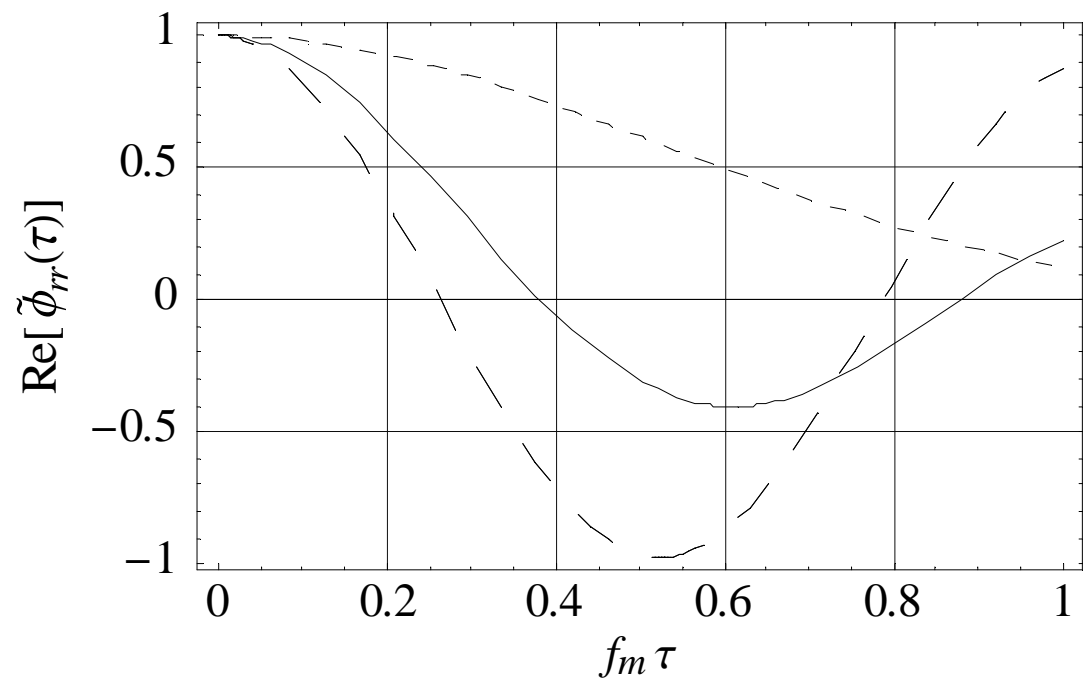

Fig. 4. Real part of the normalized autocorrelation function of the complex envelope at the mobile station due to multipath components (scatter and specular), for different urban scenarios.

Clarke's scenario: $K=0, \kappa=0$

$-\boldsymbol{Z} \cdot \mathrm{S} 1$ and $\mathrm{S} 2$ scenarios: $K=0, \kappa=10$

- - - S3 scenario: $K=0, \kappa=10$

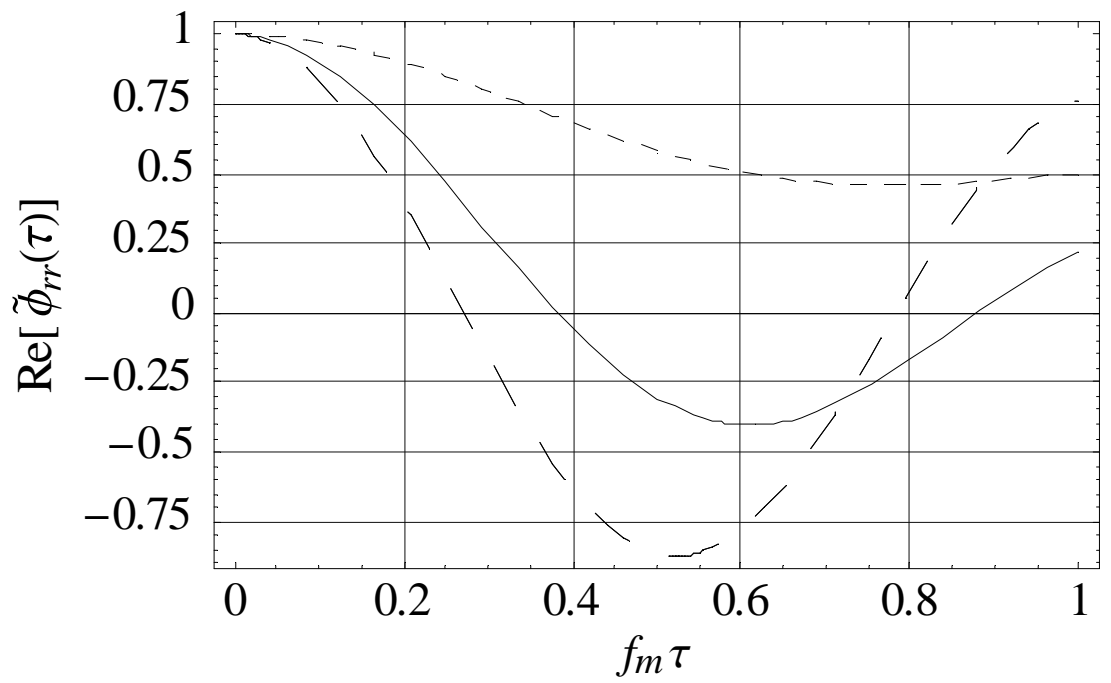

Fig. 5. Real part of the normalized autocorrelation function of the complex envelope at the mobile station due to multipath components (scatter and specular), for different urban scenarios.

Clarke's scenario: $K=0, \kappa=0$

- - - S1 and S2 scenarios: $K=1, \kappa=3, \theta_{0}=0$

- - - - S3 scenario: $K=1, \kappa=3, \theta_{0}=\pi / 2$ 


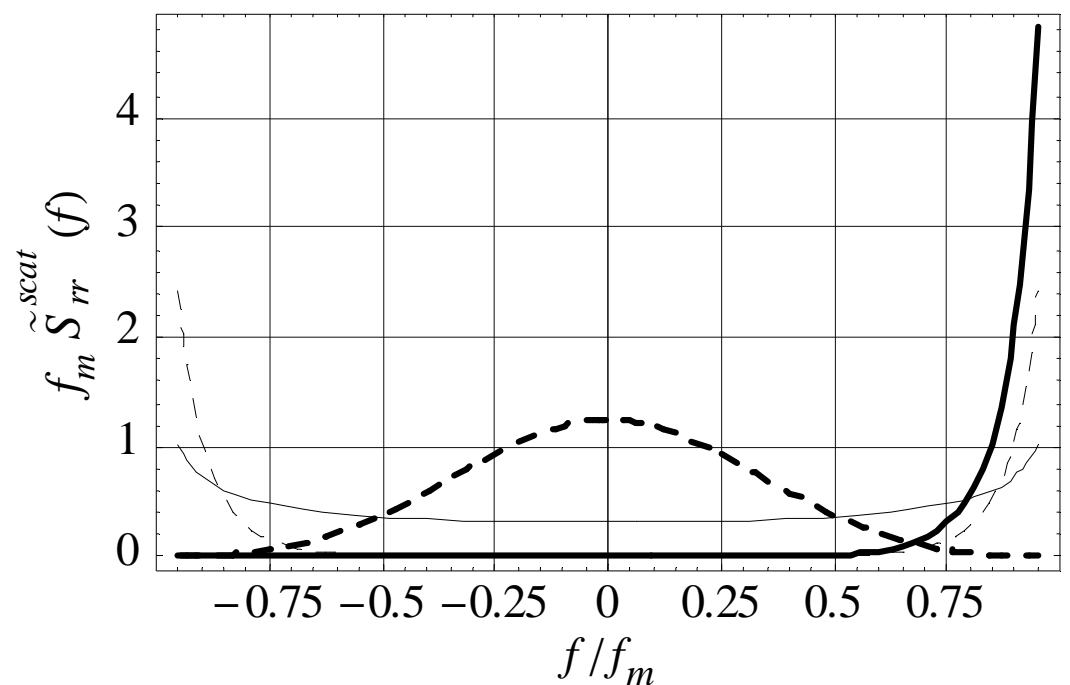

Fig. 6. Normalized power spectrum of the complex envelope at the mobile station due to scatter components, for different urban scenarios.

Clarke's scenario: $\kappa=0$

S1 scenario: $\kappa=10$

- _ . - . S2 scenario: $\kappa=10$

- - - - S3 scenario: $\kappa=10$

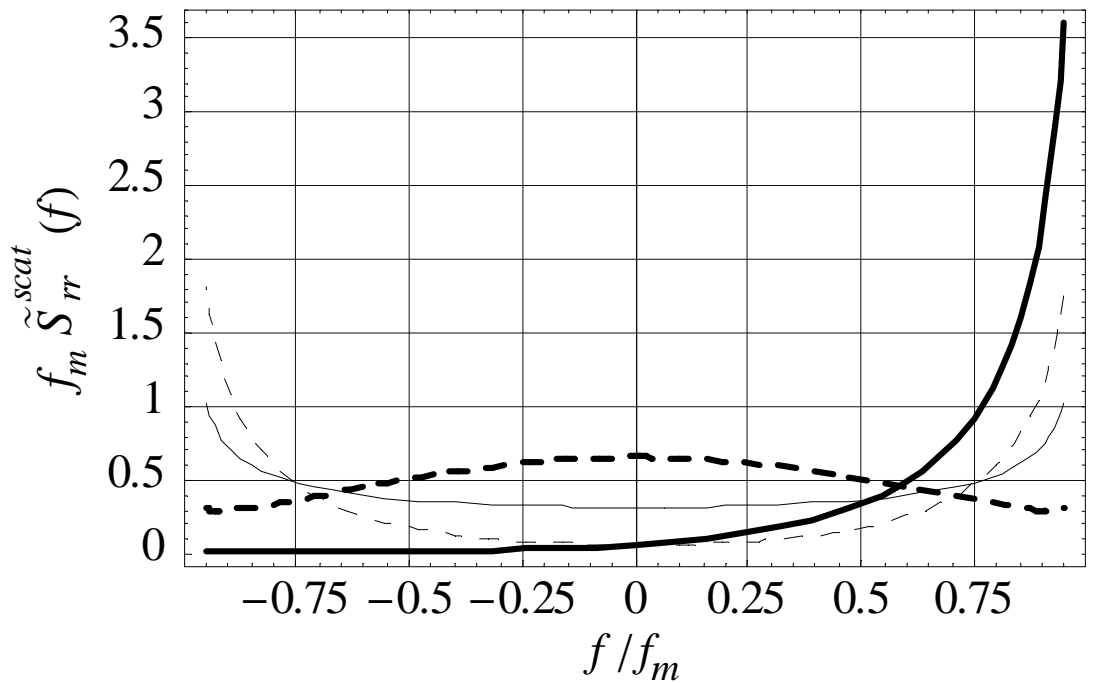

Fig. 7. Normalized power spectrum of the complex envelope at the mobile station due to scatter components, for different urban scenarios.

Clarke's scenario: $\kappa=0$

S1 scenario: $\kappa=3$

- - - - - S2 scenario: $\kappa=3$

- _ - - S3 scenario: $\kappa=3$ 


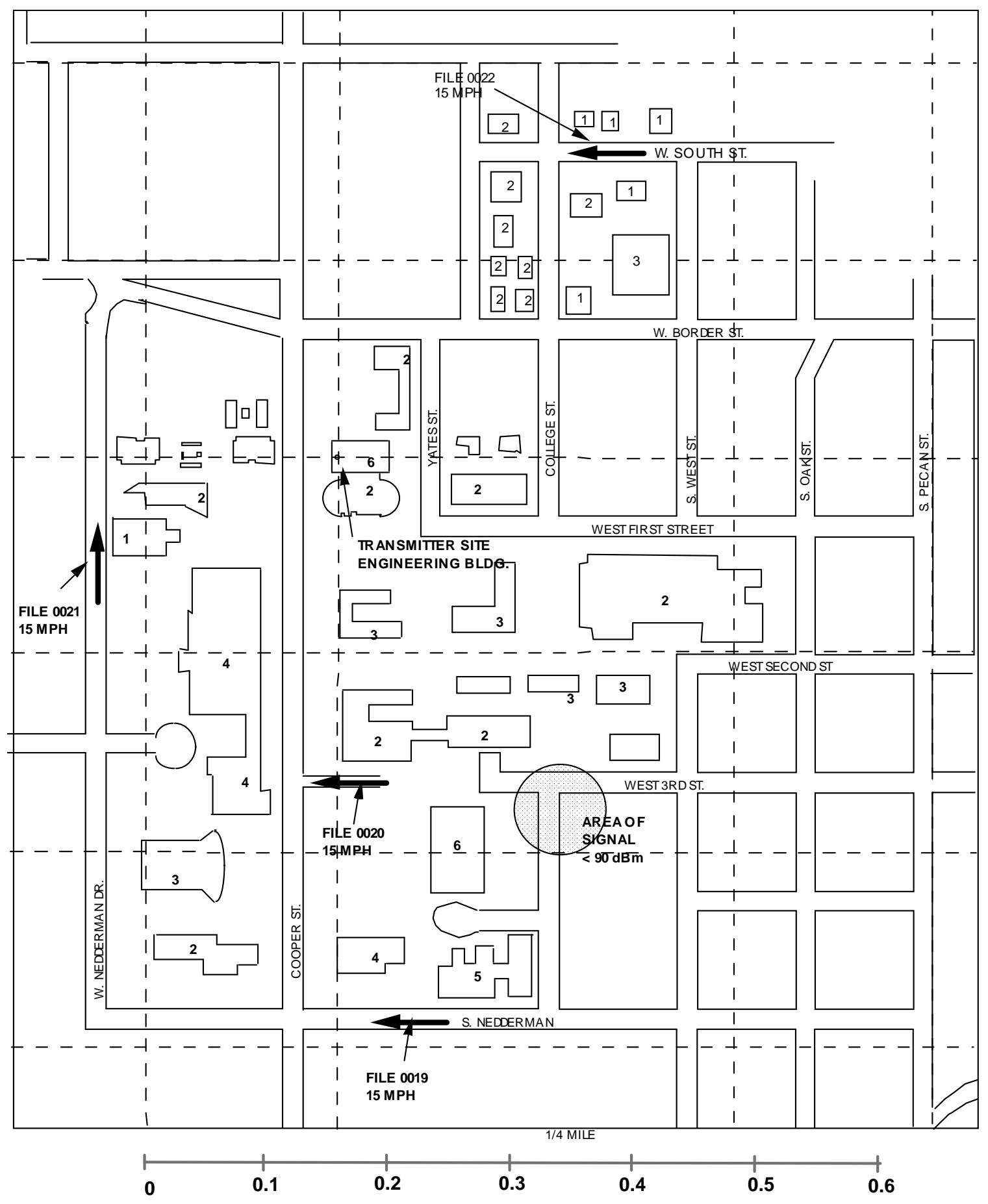

Approximate Scale in Kilometers

Fig. 8. Map of the Arlington site for collection of the urban data contained in files \#0019- \#0022. 

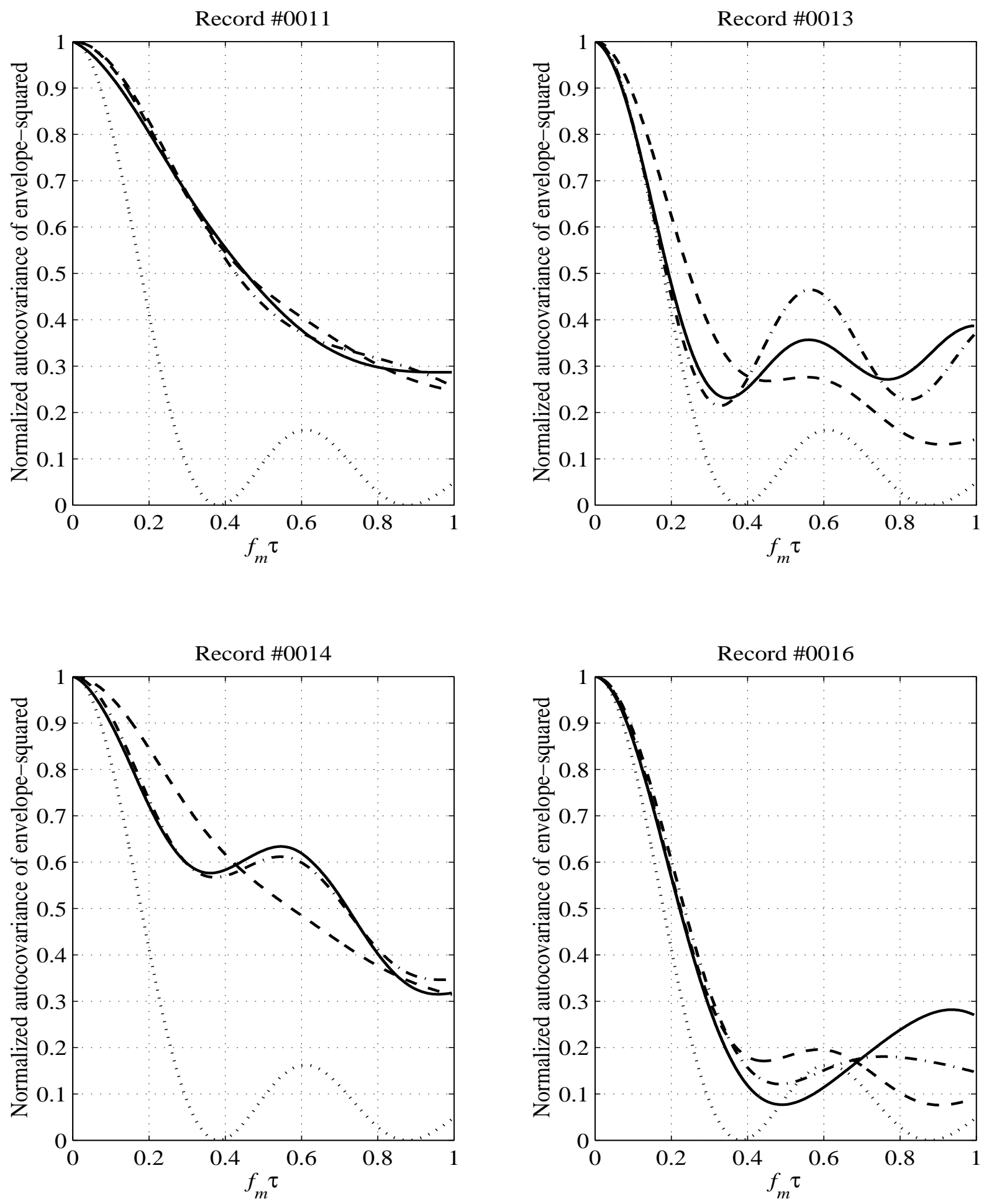

Fig. 9. Normalized autocovariance of the envelope-squared at the mobile, for different records.

- Empirical (obtained by the time-averaged estimator of the correlation function [31, p. 743])

- - - The simple model in (19), with $\left(\kappa, \theta_{p}\right)$ estimated by nonlinear least squares

- - . The composite model in $(20)$, with $\left(\kappa, \theta_{p}, \zeta\right)$ estimated by nonlinear least squares Clarke's model 

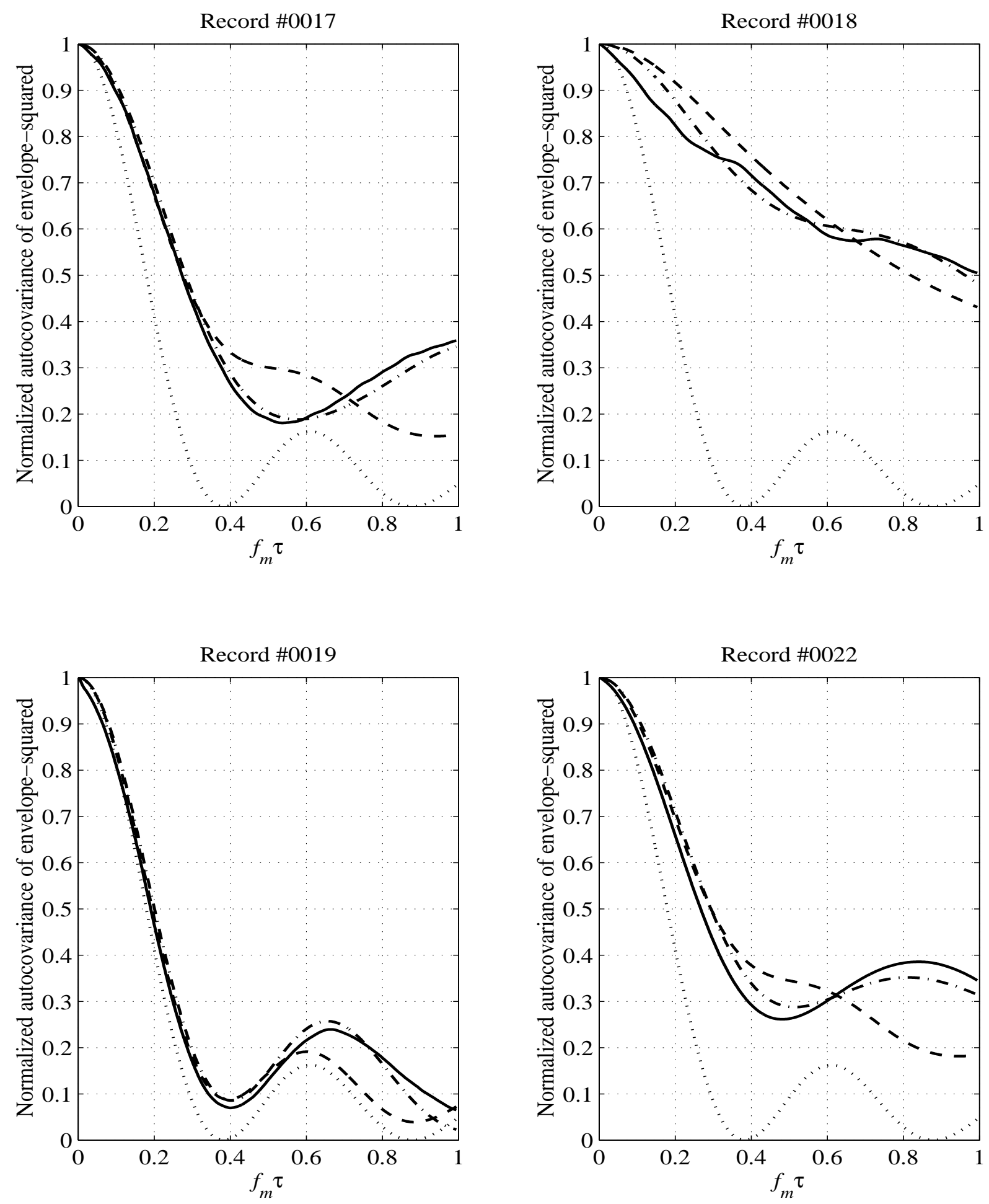

Fig. 9. Continued. 